\title{
The potential retinal hazards of curing light use for dentists
}

\author{
Nicole M Putnam ${ }^{1}$, Sara Thomas ${ }^{1}$, Grace Liao ${ }^{1}$, Emily Bennett ${ }^{1}$, Anne Breen ${ }^{1}$, Miranda Hueners ${ }^{1}$, \\ Lexi Korsten ${ }^{1}$, James Mills ${ }^{1}$, Ibrahim Mohsin ${ }^{1}$, Miles Poole ${ }^{1}$, Shelby Rookstool ${ }^{1}$, Hala Siddiqui ${ }^{1}$, \\ Kelsie Sommerfield ${ }^{1}$, Roni E Kozlowski ${ }^{1}$, John C Mitchell ${ }^{2}$, Denise Mills ${ }^{2}$ and Michael R Kozlowski ${ }^{1}$ \\ ${ }^{1}$ Arizona College of Optometry, Midwestern University, Phoenix, Arizona, United States \\ ${ }^{2}$ Midwestern University, College of Dental Medicine-Arizona, United States
}

\begin{abstract}
Background: Many recent studies have focused on the potential hazards of blue light exposures to ocular health. One group with a unique blue light exposure risk is dentists, who use curing lights that emit intense blue light during restorative procedures. During these procedures, dentists often experience brief ocular exposure to these lights. The purpose of the present study was to explore whether such exposures may have an effect on the vision and ocular health of dentists.

Methods: A group of 12 dentists who had experienced curing light exposure over a period of 10 or more years were compared to a group of eight control subjects with no such exposure. The subjects were tested for visual acuity and contrast sensitivity. Their retinas were examined using fundus imaging and optical coherence tomography. Macular pigment optical density was measured. The likelihood that brief blue light exposure could lead to ocular effects was further explored by subjecting a retinal pigment epithelial cell (RPE) line to such exposures.

Results: Although no visual defects or ocular pathologies were found in either group, the dentist group differed from the control group in having increased macular thickness $(P<0.02)$, a higher incidence of macular vessel tortuosity $(P<0.05)$, and greater variance in their macular pigment optical density values $(P<0.01)$. RPE cells that received blue light exposure similar to those sustained by dentists demonstrated a change in physiology.

Conclusions: Retinal changes were found in dentists, which, while not pathological in themselves, are associated with some retinal pathologies. Further studies are necessary to determine whether these signs correlate with the degree of curing light exposure and to determine whether they eventually develop into pathological conditions.

\section{KEYWORDS}

blue light, curing lights, macular changes, contrast sensitivity, macular pigment optical density, retinal thickness, retinal pigment epithelial cells, retinal pigment epithelium, epiretinal membrane, age-related macular degeneration
\end{abstract}

Correspondence: Michael R Kozlowski, Professor of Optometry, Midwestern University / AZCOPT, 19555 North 59th Avenue, Glendale, AZ 85308, Fax: 623-572-3911. Email: mkozlo@midwestern.edu. ORCID iD: https://orcid.org/0000-0003-1213-7015

How to cite this article: Putnam NM, Thomas S, Liao G, Bennett E, Breen A, Hueners M, Korsten L, Mills J, Mohsin I, Poole M, Rookstool S, Siddiqui H, Sommerfield K, Kozlowski RE, Mitchell JC, Mills D, Kozlowski MR. The potential retinal hazards of curing light use for dentists. Med Hypothesis Discov Innov Optom. 2021 Summer; 2(2): 77-84. https://doi.org/10.51329/mehdioptometry129

Received: 16 September 2021; Accepted: 11 November 2021

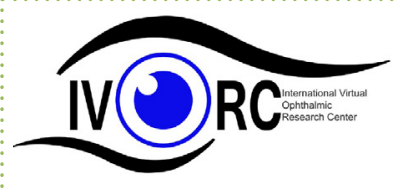

Copyright (C) Author(s). This is an open-access article distributed under the terms of the Creative Commons Attribution-NonCommercial 4.0 International License (http://creativecommons.org/licenses/by-nc/4.0/) which permits copy and redistribute the material just in noncommercial usages, provided the original work is properly cited. (c) (i) (s) 


\section{INTRODUCTION}

Resin-based materials are commonly used in dentistry for restorative procedures, such as the repair of cracked or chipped teeth or dental fillings [1,2]. Many of these resins are cured by exposure to blue light [1]. Light is produced by hand-held devices that can emit blue light at intensities exceeding 1 watt per centimeter squared $\left(\mathrm{W} / \mathrm{cm}^{2}\right)$. A common practice among dentists is to view the area being treated briefly when the curing light is first activated, to ensure that the light is properly positioned, and then to either look away or view through blue light filtering lenses during the remainder of the exposure period [1]. This practice can lead to repetitive, brief exposure to high-intensity blue light.

Numerous laboratory studies have demonstrated that exposure to blue light can damage elements of the retina, including retinal pigment epithelium (RPE) cells [3-10]. Damage to cultured RPE cells can occur at blue light intensities in the $0.2-20-\mathrm{mW} / \mathrm{cm}^{2}$ range $[4,7,10,11]$. The prospect of damage to RPE cells resulting from blue light exposure is concerning, because dysfunction of these cells is thought to contribute to the pathology of several ocular disorders, including age-related macular degeneration (AMD) [12, 13]. The relationship between blue light exposure and AMD is supported by population-based studies, which found that higher levels of exposure to sunlight can increase the incidence and severity of AMD [14-17]. This harmful effect of sunlight is almost certainly due to its blue light component, since light in this wavelength range is the most damaging to cells of the retina $[3-7,11,18,19]$.

Unlike the short exposure that occurs in dental practice, however, the blue light exposures experienced in laboratory experiments, and even more so in population-based studies, generally lasted for hours and were sometimes repeated over many years [3-11, 14-19]. This difference in exposure conditions makes it difficult to generalize the consequences of blue light exposure in these studies [3-11, 14-19] to what might occur after repeated, brief exposures to curing lights. However, it is possible that the curing light exposure experienced by dentists during restorative dental procedures, although brief, is sufficient to produce detrimental ocular effects.

To explore this possibility, the present study compared the vision and ocular health of dentists who have experienced brief exposure to intense blue light over a period of years to those of individuals with no such exposures. In addition, it examined whether brief, repeated blue light exposure can affect the functioning of retinal cells in vitro, using an RPE cell line culture.

\section{METHODS}

This case-control and in vitro study was approved by the Midwestern University Institutional Review Board. All subjects gave their informed consent for participation. The subjects were divided into a dentist group ( $\mathrm{n}=$ 12 ) and a non-dentist control group $(n=8)$. A survey was utilized to determine curing light exposure, ocular health, and patient demographics. The dentist group reported experiencing $0.5-45$ brief blue light exposure events from curing lights per month (mean \pm standard deviation for the number of blue light exposures $=10.9$ \pm 16.4 ) compared to the control group, who experienced no curing light exposures. Prospective subjects with less than 10 years of dental practice (for the dentist group only); retinal diseases, such as glaucoma, AMD, or diabetic retinopathy; cataracts, with the exception of trace nuclear sclerotic cataracts; and use of high ocularrisk drugs, such as Plaquenil ${ }^{\mathrm{Ts}}$, were excluded from the study.

Best-corrected distance visual acuity (BCDVA) was measured using either a Snellen or Early Treatment Diabetic Retinopathy Study (ETDRS) chart, and contrast sensitivity (CS) was measured using a Mars Letter Contrast Sensitivity Chart (Mars Perceptrix Corporation, Chappaqua, NY). ETDRS acuity was converted to Snellen acuity before analysis [20]. Macular pigment optical density (MPOD) was measured using a QuantifEye MPS II ${ }^{\mathrm{Tm}}$ instrument (Zea Vision, Chesterfield, MO, USA).

Optical coherence tomography (OCT) was performed using a Spectralis HRA + OCT ${ }^{\mathrm{m}}$ (Heidelberg, Germany). To reduce variability in the results as well as possible investigator bias, all persons performing OCT scans received the same training in the operation of the instrument, performed scans on both dentists and controls, and were blinded to any measurements made from their scans until all images were analyzed. The thickness of the central subfield (SCF) (i.e., a 1-mm-diameter area centered on the fovea) was measured automatically during OCT. The scans were then examined independently by two optometrists (MRK and ST) to identify any gross retinal abnormalities or defects.

Retinal images were captured using either the OCT instrument, a Daytona ${ }^{\mathrm{\tau}}$ ultrawide-field imager (Optos, Dunfermline, UK) or a TRC-NW400 fundus camera (Topcon, Tokyo, Japan). Optos imagers and 
OCT instruments produce en face macular images at least as good as those produced by fundus cameras in terms of imaging inner retinal pathology [21,22] All operators of each instrument received the same training, and images of both dentist and control retinas were captured on all instruments. The images were then examined independently by two optometrists (MRK and ST) to identify any gross retinal abnormalities or defects.

The blood vessel course was assessed in an area within two optic nerve diameters of the fovea, using fundus photographs and en face displays of OCT scans. The images were rated for increased blood vessel tortuosity by an optometrist (MRK) skilled in viewing such images, and who was blinded to group allocation. An image was graded as having increased tortuosity if one or more vessels contained at least four arcs with a sagitta equal to or greater than the diameter of a retinal vein at the optic nerve head from the same image. For statistical analysis, each subject was assigned a value of 0,1 , or 2 , depending on whether increased tortuosity was seen in no retina, one retina, or both retinas, respectively.

Effects of blue light exposure on RPE cells were investigated. Cells of a human RPE cell line (ARPE-19; American Type Culture Collection [ATCC], Manassas, VA) were cultured to confluence in Dulbecco's modified Eagle's medium (ATCC) with 1\% fetal bovine serum in T25 flasks for 1 month. These culture conditions promote the development of an RPE-like morphology [23, 24]. The flasks were then exposed to blue light from an UltraLume 5 LED $^{\circledR}$ dental curing light (Ultradent Products Inc., South Jordan, UT) from Monday through Friday, over a period of 4 weeks. Each area of the flask was exposed to blue light at an intensity of $2 \mathrm{~mW} / \mathrm{cm}^{2}$ for approximately $0.5 \mathrm{~s}$ to simulate the brief exposures experienced by dentist during curing procedures. Two flasks were given no exposure (control) and one each was given 1, 3, or 10 exposures, spread evenly over a 1-hour period, each day. At the end of the 4-week exposure period, the cells were re-plated in triplicate and were stained for senescence-associated beta-galactosidase (SABG) activity, using a commercial kit (Fisher Scientific, Waltham, MA). A Moticam 5 digital camera ${ }^{\mathrm{TM}}$ (Fisher Scientific) attached to a Motic ${ }^{\mathrm{TM}}$ inverted microscope (Fisher Scientific) was used to photograph the cultures in the T-25 flasks before they were dissociated and to capture one image from the same location in each of the triplicate plates of the re-plated cells. These images contained 29-125 cells. An optical density quantification program (GelAnalyzer ${ }^{\oplus}$ ) was used to measure the optical density of the SABG-staining of each cell in these images.

Analyses were performed using IBM ${ }^{\mathrm{TM}}$ SPSS $^{\mathrm{TM}}$ Statistics for Windows, version 27.0 (IBM Corp., Armonk, N.Y., USA). Individual mean values were compared using $t$-tests. Group data were compared using analysis of variance (ANOVA), and variances were compared using an F-test. The predetermined significance level for rejection of the null hypothesis (alpha) was 0.05 .

\section{RESULTS}

The average age of subjects in the dentist group (mean $\pm \mathrm{SD}, 62 \pm 4$ years) was not significantly different from that of subjects in the control group (mean $\pm \mathrm{SD}, 58 \pm 5$ years; $t$-test, $P>0.05$ ). The measurement of BCDVA also revealed no significant difference between the dentist and control groups (mean $\pm \mathrm{SD}, 20 / 16 \pm 4$ and $20 / 16 \pm 2$, respectively; Snellen notation, $t$-test, $P>0.05$ ), and contrast sensitivity also did not differ between the two groups (mean $\pm \mathrm{SD}, 1.75 \pm 3$ and $1.74 \pm 5$ cycles per degree, respectively; $t$-test, $P>0.05$ ). In addition, OCT scans and fundus photographs revealed no pathologies in the retinas of either group.

However, OCT scans revealed a difference in the average thickness of the central subfield (CSF) of the retina between the two groups. Although the values for both groups were within a standard deviation of the reported CSF thickness in healthy eyes (mean \pm SD, $271.4 \pm 19.6 \mu \mathrm{m}$ ) [25], the CSF thickness of the dentist group (mean $\pm S D, 285.3 \pm 21.7 \mu \mathrm{m})$ was significantly greater $(t$-test, $P<0.02)$ than that of the of the control group $(254.4 \pm 33.3 \mu \mathrm{m}$; Figure 1$)$. Within the dentist group, there was no correlation between CSF thickness and the monthly frequency of exposure to curing light (ANOVA, $P>0.05$ ). Blood vessel tortuosity also differed significantly between the two groups $(t$-test, $P<0.05)$, with nine of the 12 subjects in the dentist group meeting the criterion for tortuosity in at least one macula, as compared to only two of eight subjects in the control group (Figure 2).

A further difference between the two groups was noted during the measurement of MPOD. The variance in the MPOD values was found to be significantly greater in the dentist group than in the control group ( $F$ test, $P<0.01$, Figure 3). On the other hand, the mean MPOD values for dentists (mean \pm SD, $0.46 \pm 0.25$ density units) and controls (mean $\pm \mathrm{SD}, 0.35 \pm 0.09$ density units) were not significantly different $(t$-test, $P>0.05)$, and there was no significant relationship between MPOD and the reported number of curing light exposures per month in the dentist group (ANOVA, $P>0.05$ ). 


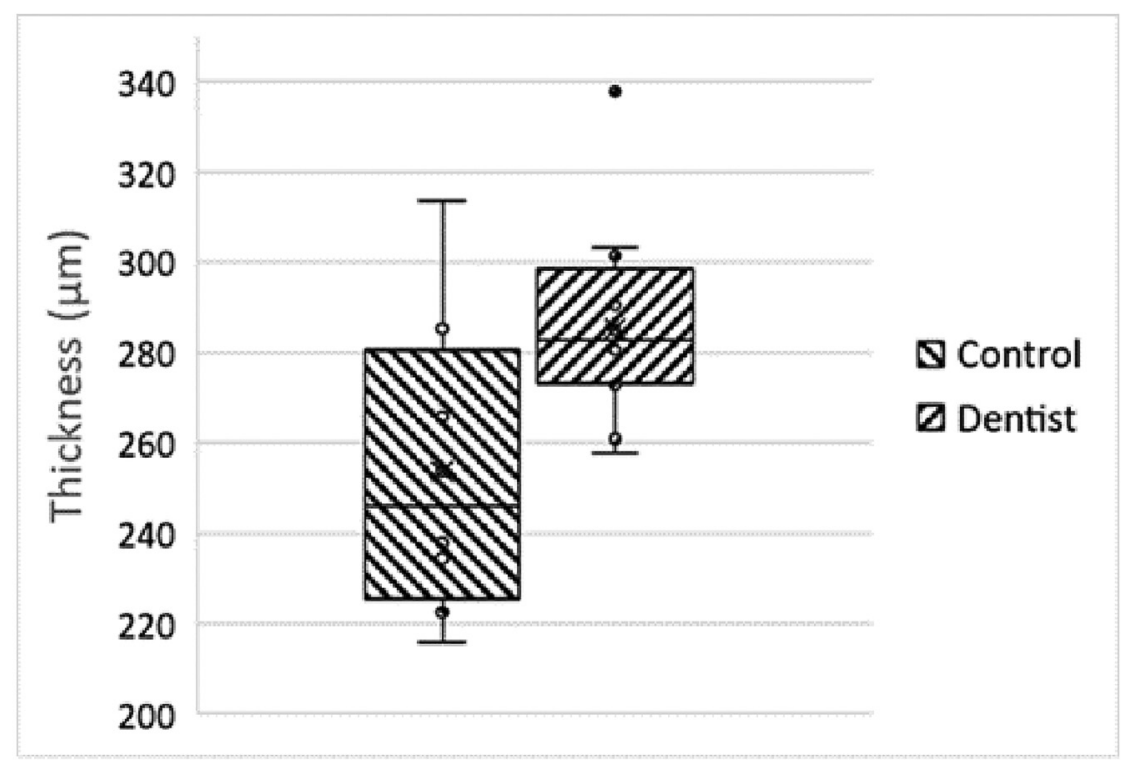

Figure 1. Macular thickness, measured by optical coherence tomography (OCT) as the central subfield. Boxes enclose the second and third quartiles. The average macular thickness of the dentist group was significantly greater than that of the control group $(t$-test, $P$-value $<0.02)$. The horizontal line in each box represents the median value and " $X$ " represents the mean value. Error bars indicate standard error.
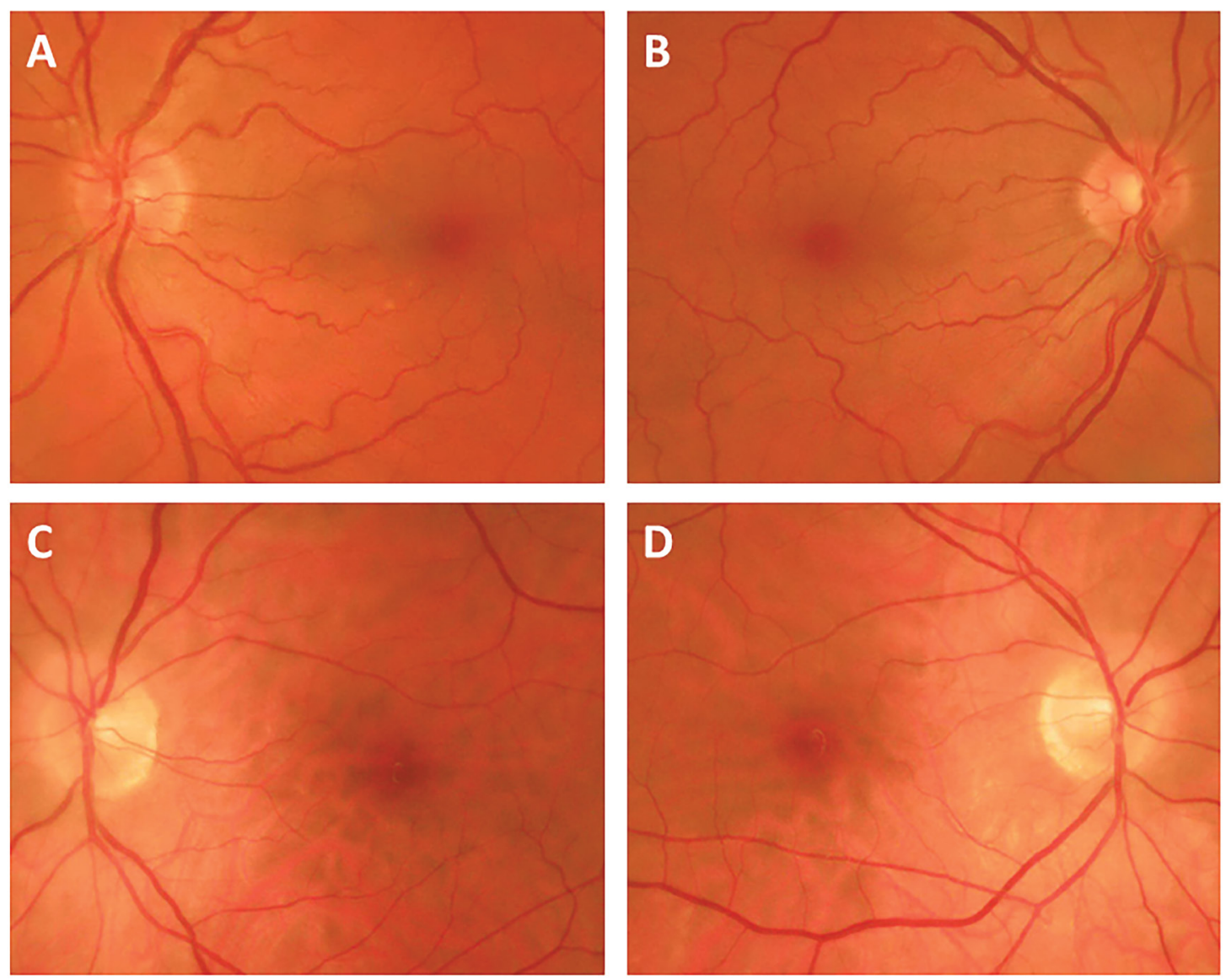

Figure 2. Examples of the fundus of a dentist who met the criteria for blood vessel tortuosity (A and $B$ ) and that of a control subject who did not (C and D). 


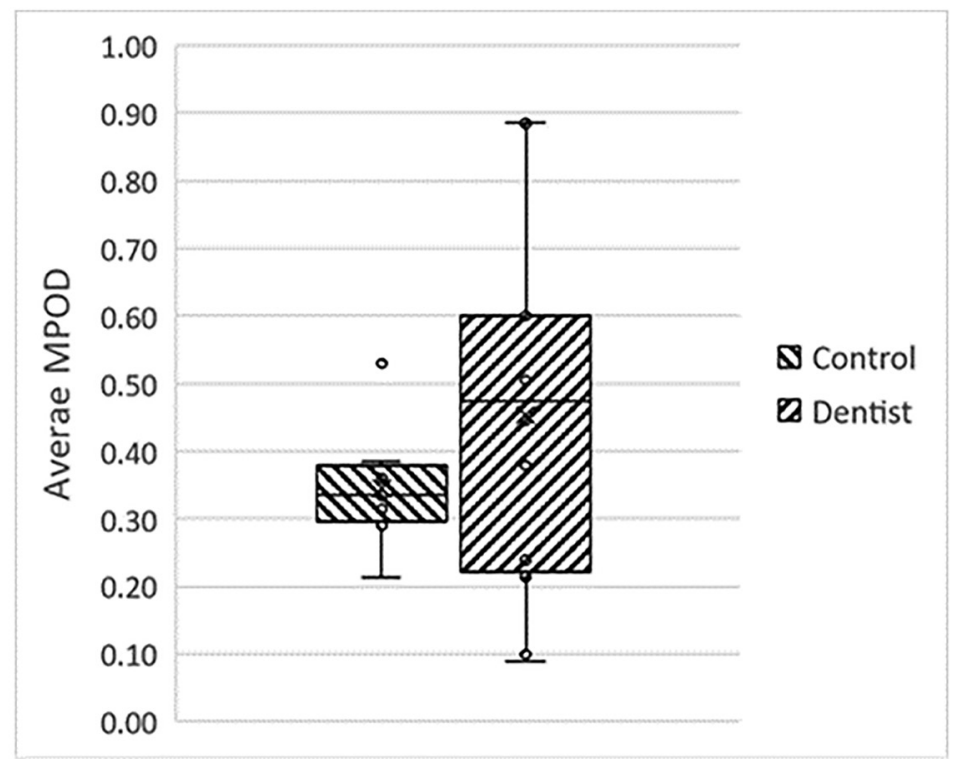

Figure 3. Comparison of Macular pigment optical density or MPOD (average of both eyes) between subjects in the dentist group and in the control group. Graph parameters are as shown in Figure 1.

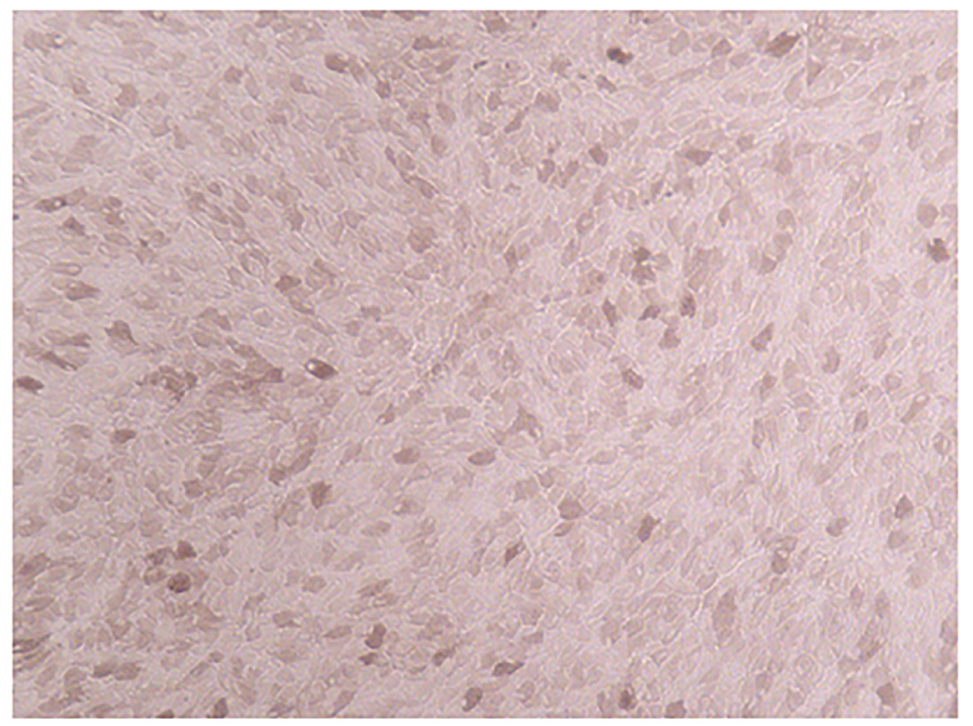

Figure 4. A typical ARPE-19 cell layer not treated with blue light before dissociation and staining for senescence associated betagalactosidase (SABG). Cells show a cobblestone-like arrangement and are beginning to produce pigment.

Effect of brief blue light exposure on RPE cells was studied as follows. ARPE-19 cells cultured in T25 flasks formed continuous monolayers and developed cobblestone-like arrangements and pigmentation, as previously reported (Figure 4) [26]. When the layers were dissociated and the cells were immediately replated for SABG-staining, the cells displayed a moderate amount of staining, as previously reported (Figure $5 A)[27]$.

The cells dissociated from layers that had been subjected to short, daily exposures to blue light $\left(2 \mathrm{~mW} / \mathrm{cm}^{2}\right)$ for 4 weeks showed no significant change in their levels of SABG staining as compared to unexposed cells when 1 or 3 exposures were given per day. However, when 10 exposures were administered per day, the density of the SABG staining was reduced by $11 \%$ after 4 weeks ( 1 -tailed $t$-test, $P<0.05$ ) (Figure 5 ). 

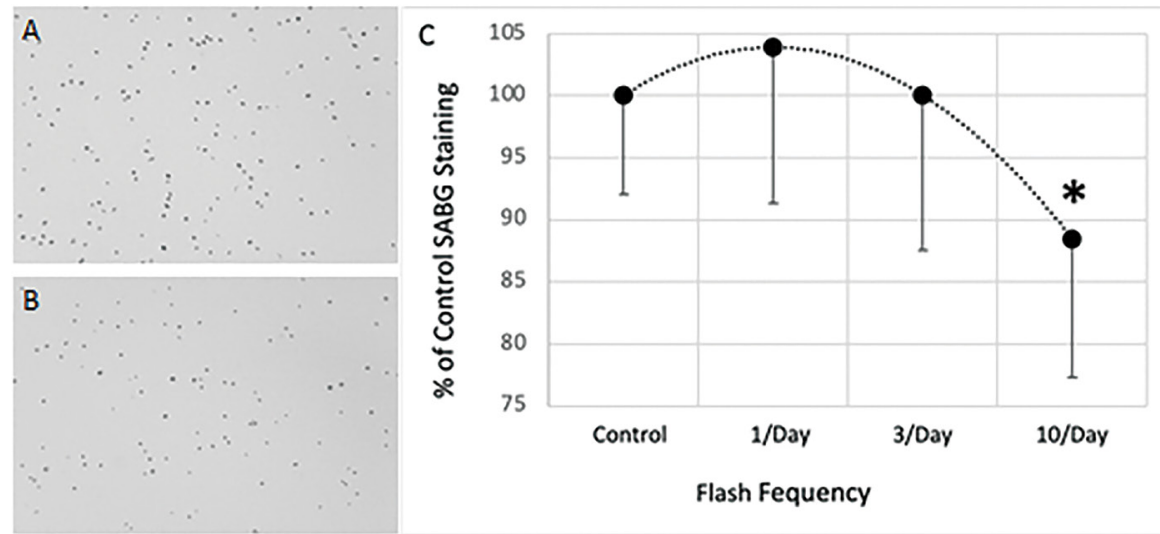

Figure 5. Effect of repeated, short exposures to a curing light on the physiology of retinal pigment epithelium (RPE) cells. (A) Senescence associated beta-galacosidase (SABG)-staining of cells dissociated from RPE layers that had not been exposed to flashes of blue light. (B) SABG-staining in cells exposed to 10 curing light flashes per day for 4 weeks. (C) Level of SABG-staining as a function of the number of blue light exposures per day, over a period of 4 weeks. The asterisk indicates a significant difference from the level of untreated cells $(1$-tailed $t$-test, $P<0.05)$.

\section{DISCUSSION}

The present study found that dentists who use curing lights in their practice demonstrate changes in their retinal organization. These changes consisted of thickening of the macular CSF region, increased blood vessel tortuosity, and higher variability in MPOD. These changes may be due to curing light exposure, since, during a dental procedure, the radiation from curing lights sometimes shine into the dentist's eye either directly or by reflection from a bright surface, such as tooth enamel. Curing lights, such as the UltraLume 5 LED $^{\circledR}$ curing light used in the present study, emit blue wavelength light at high intensities $[1,2,28]$. These intensities exceed those reported to cause damage to retinal cells in experimental studies $[4,7,10,11]$. The durations of blue light exposure experienced by dentists are, however, much shorter than those used in experimental studies, which may mitigate such damage.

To investigate whether brief exposure to blue light might affect the functioning of retinal cells, ARPE-19 cell layers were subjected to short periods of blue light exposure at an intensity in the range that has been shown to affect RPE cell physiology with prolonged exposure $[4,5,7,29]$. Although there is some question regarding the validity of ARPE-19 cells as a model for native RPE cells, these cells appear to be a suitable model when cultured under the appropriate conditions [30]. The length of the ARPE-19 cell exposure in this study was chosen to mimic that of unintentional curing light exposure during dental procedures. Brief exposure of ARPE-19 cells to blue light reduced their SABG activity, similar to that observed with prolonged exposure [29]. Reduced SABG staining may be due to a decrease in lysosomal activity [31]. This result is consistent with the concept that even brief exposures to curing light radiation, such as experienced by dentists, could be hazardous to the retina.

Further evidence of a potential retinal hazard of curing lights use was found by comparing the vision and retinal health of dentists who had experienced repeated blue light exposure and those of control subjects. These comparisons revealed no evidence of an effect of blue light exposure on BCDVA or on contrast sensitivity. Likewise, the average MPOD values did not differ between the two groups. However, there were increases in macular CSF thickness, macular blood vessel tortuosity, and MPOD variability in the retinas of the dentists compared to that of the controls. Increases in macular CSF thickness and blood vessel tortuosity, such as that observed in the dentist group in this study, are also seen in the development of epiretinal membranes (ERMs) $[32,33]$. However, no other signs of ERMs, such as increased macular reflectance, changes in the topography of the foveal pit, reduced contrast sensitivity, or reduced MPOD, were observed in the present study [32, 34, 35]. Similarly, although the broader range of MPOD values in the dentist group than in the control group was consistent with a disturbance of macular pigmentation, other evidence of a disruption of macular pigmentation, such as the mottling associated with AMD, was not observed [36].

We investigated whether one of the changes seen in the dentist group, i.e., increased CSF thickness, correlated with the frequency of blue light exposure, but found no relationship. This raises the possibility that the retinal changes seen in the dentist group were not due to blue light exposure, but rather to some other element in the practice of dentistry. On the other hand, the reporting of blue light exposure by dentists was only an estimate 
and may, therefore, have been somewhat inaccurate. In addition, the frequency, rather than the number of exposures, was used to investigate the correlation. Increased CSF thickness may have a better correlation with total exposures. Future studies are needed to quantitate the amount of blue light exposure from curing light experienced by dentists more carefully and to determine whether there is a correlation between the level of exposure and retinal changes, such as those seen in the present study.

\section{CONCLUSIONS}

The present study did not find any visual defects or retinal pathologies in the group of dentists, nor did it establish that the retinal changes that were seen in the dentist group were the result of curing light exposure. Even so, the fact that retinal changes were found in the dentist group is concerning, particularly since these changes resemble signs that occur in some types of ocular pathologies. In addition, the finding that brief exposure to blue light can affect the functioning of retinal cells raises the possibility that these changes seen in dentists may be related to curing light exposure. Further studies with more thorough accounting of the frequency and number of curing light exposures will be needed to verify the relationship between such exposures and the occurrence of the retinal changes seen in this study. In addition, dentists exposed to curing light will need to be followed for a longer period of time to determine whether the retinal signs seen in this study develop into frank retinal pathologies.

\section{ETHICAL DECLARATION}

Ethical approval: This study was approved by the Midwestern University Institutional Review Board. All subjects gave their informed consent for participation.

\section{Conflict of interests: None}

\section{FUNDING}

None.

\section{ACKNOWLEGMENTS}

None.

\section{REFERENCES}

1. Brucia JJ (2020). 'Light Curing in Restorative Dentistry: The Devil Is in the Details'. Dentistry Today. Available at: https://www. dentistrytoday.com/light-curing-in-restorative-dentistry-the-devil-is-in-the-details/(Accessed: Oct 01, 2021)

2. Rueggeberg FA, Giannini M, Arrais CAG, Price RBT. Light curing in dentistry and clinical implications: a literature review. Braz Oral Res. 2017;31(suppl 1):e61. doi: 10.1590/1807-3107BOR-2017.vol31.0061 pmid: 28902241

3. Sparrow JR, Cai B. Blue light-induced apoptosis of A2E-containing RPE: involvement of caspase-3 and protection by Bcl-2. Invest Ophthalmol Vis Sci. 2001;42(6):1356-62. pmid: 11328751

4. Chu R, Zheng X, Chen D, Hu DN. Blue light irradiation inhibits the production of HGF by human retinal pigment epithelium cells in vitro. Photochem Photobiol. 2006;82(5):1247-50. doi: 10.1562/2006-04-19-RA-880 pmid: 16740060

5. Roehlecke C, Schaller A, Knels L, Funk RH. The influence of sublethal blue light exposure on human RPE cells. Mol Vis. 2009;15:1929-38. pmid: 19784391

6. Arnault E, Barrau C, Nanteau C, Gondouin P, Bigot K, et al. Phototoxic Action Spectrum on a Retinal Pigment Epithelium Model of Age-Related Macular Degeneration Exposed to Sunlight Normalized Conditions. PLoS ONE. 2013;8(8):e71398. doi: 10.1371/journal.pone. 0071398 pmid: 24058402

7. Chamorro E, Bonnin-Arias C, Pérez-Carrasco MJ, de Luna JM, Vázquez D, Sánchez-Ramos C. Effects of Light-emitting Diode Radiations on Human Retinal Pigment Epithelial CellsIn Vitro. Photochemistry and Photobiology. 2013;89(2):468-73. doi: 10.1111/j.1751-1097.2012.01237.x pmid: 22989198

8. Lin C-H, Wu M-R, Li C-H, Cheng H-W, Huang S-H, Tsai C-H, et al. Editor's Highlight: Periodic Exposure to Smartphone-Mimic Low-Luminance Blue Light Induces Retina Damage Through Bcl-2/BAX-Dependent Apoptosis. Toxicological Sciences. 2017;157(1):196-210. doi: 10.1093/toxsci/kfx030 pmid: 28184904

9. Kuse Y, Ogawa K, Tsuruma K, Shimazawa M, Hara H. Damage of photoreceptor-derived cells in culture induced by light emitting diode-derived blue light. Scientific Reports. 2014;4:5223. doi: 10.1038/srep05223 pmid: 24909301

10. Kozlowski MR, Putnam N, Kozlowski RE, Baker J. (2019). 'Is there a safe level of blue light exposure?' Annual meeting of the American Academy of Optometry. Available at: https://www.aaopt.org/detail/knowledge-base-article/is-there-a-safe-level-of-blue-lightexposure-339195-3238625 (Accessed: Oct 01, 2021)

11. Kozlowski MR. Blue light-induced retinal damage: a brief review and a proposal for examining the hypothetical causal link between person digital device use and retinal injury. Medical Hypothesis, Discovery \& Innovation in Optometry. 2020;1(3):129-34. doi: $10.51329 /$ mehdioptometry118

12. R. Sparrrow J, Hicks D, P. Hamel C. The Retinal Pigment Epithelium in Health and Disease. Current Molecular Medicine. 
2010;10(9):802-23. doi: 10.2174/156652410793937813 pmid: 21091424

13. Bird A. Role of retinal pigment epithelium in age-related macular disease: a systematic review. Br J Ophthalmol. 2021;105(11):14691474. doi: 10.1136/bjophthalmol-2020-317447 pmid: 32950958

14. Hunter JJ, Morgan JIW, Merigan WH, Sliney DH, Sparrow JR, Williams DR. The susceptibility of the retina to photochemical damage from visible light. Progress in Retinal and Eye Research. 2012;31(1):28-42. doi: 10.1016/j.preteyeres.2011.11.001 pmid: 22085795

15. Margrain TH, Boulton M, Marshall J, Sliney DH. Do blue light filters confer protection against age-related macular degeneration? Progress in Retinal and Eye Research. 2004;23(5):523-31. doi: 10.1016/j.preteyeres.2004.05.001 pmid: 15302349

16. Tomany SC, Cruickshanks KJ, Klein R, Klein BE, Knudtson MD. Sunlight and the 10-year incidence of age-related maculopathy: the Beaver Dam Eye Study. Arch Ophthalmol. 2004;122(5):750-7. doi: 10.1001/archopht.122.5.750 Erratum in: Arch Ophthalmol. 2005;123(3):362. pmid: 15136324

17. Schick T, Ersoy L, Lechanteur YTE, Saksens NTM, Hoyng CB, den Hollander AI, et al. History of Sunlight Exposure Is a Risk Factor for Age-Related Macular Degeneration. Retina. 2016;36(4):787-90. doi: 10.1097/IAE.0000000000000756 pmid: 26441265

18. Algvere PV, Marshall J, Seregard S. Age-related maculopathy and the impact of blue light hazard. Acta Ophthalmologica Scandinavica. 2006;84(1):4-15. doi: 10.1111/j.1600-0420.2005.00627.x pmid: 16445433

19. Sui G-Y, Liu G-C, Liu G-Y, Gao Y-Y, Deng Y, Wang W-Y, et al. Is sunlight exposure a risk factor for age-related macular degeneration? A systematic review and meta-analysis. British Journal of Ophthalmology. 2013;97(4):389-94. doi: 10.1136/bjophthalmol-2012-302281 pmid: 23143904

20. Kaiser PK. Prospective evaluation of visual acuity assessment: a comparison of snellen versus ETDRS charts in clinical practice (An AOS Thesis). Trans Am Ophthalmol Soc. 2009;107:311-24. pmid: 20126505

21. Byberg S, Vistisen D, Diaz L, Charles MH, Hajari JN, Valerius M, et al. Optos wide-field imaging versus conventional camera imaging in Danish patients with type 2 diabetes. Acta Ophthalmologica. 2019;97(8):815-20. doi: 10.1111/aos.14118 pmid: 30985086

22. Riazi-Esfahani H, Khalili Pour E, Fadakar K, Ebrahimiadib N, Ghassemi F, Nourinia R, et al. Multimodal imaging for paracentral acute maculopathy; the diagnostic role of en face OCT. International Journal of Retina and Vitreous. 2021;7(1):13. doi: 10.1186/s40942021-00283-y pmid: 33593444

23. Samuel W, Jaworski C, Postnikova OA, Kutty RK, Duncan T, Tan LX, et al. Appropriately differentiated ARPE-19 cells regain phenotype and gene expression profiles similar to those of native RPE cells. Mol Vis. 2017;23:60-89 pmid: 28356702

24. Pao P-J, Emri E, Abdirahman SB, Soorma T, Zeng H-H, Hauck SM, et al. The effects of zinc supplementation on primary human retinal pigment epithelium. Journal of Trace Elements in Medicine and Biology. 2018;49:184-191. doi: 10.1016/j.jtemb.2018.02.028 pmid: 29523386

25. Chan A, Duker JS, Ko TH, Fujimoto JG, Schuman JS. Normal Macular Thickness Measurements in Healthy Eyes Using Stratus Optical Coherence Tomography. Archives of Ophthalmology. 2006;124(2):193-8. doi: 10.1001/archopht.124.2.193 pmid: 16476888

26. Kozlowski MR, Putnam N, Anderson M, Kozlowski RE, Baker J. (2017). 'Cumulative Effects of Low Intensity Blue Light Exposure on the Functioning of Cultured RPE Cells'. Annual meeting of the American Academy of Optometry. Available at: https://www.aaopt. org/detail/knowledge-base-article/cumulative-effects-of-low-intensity-blue-light-exposure-on-the-functioning-of-cultured-rpe-cells (Accessed: Oct 01, 2021)

27. Severino J, Allen RG, Balin S, Balin A, Cristofalo VJ. Is beta-galactosidase staining a marker of senescence in vitro and in vivo? Exp Cell Res. 2000;257(1):162-71. doi: 10.1006/excr.2000.4875 pmid: 10854064

28. Price RB, Felix CA, Andreou P. Evaluation of a dual peak third generation LED curing light. Compend Contin Educ Dent. 2005;26(5):331-2, 334, 336-8 passim; quiz 348. pmid: 15892221

29. Steinshouer N, Bird Z, Chmielewski J, Meske J, Baker J, Kozlowski RE, et al. (2016). ‘Effects of an Environmentally Relevant Level of Blue Light Exposure on Cultured RPE Cells' Annual meeting of the American Academy of Optometry. Available at: https://www. aaopt.org/detail/knowledge-base-article/effects-environmentally-relevant-level-blue-light-exposure-cultured-rpe-cells (Accessed: Oct 01,2021 )

30. Kozlowski MR. The ARPE-19 cell line: mortality status and utility in macular degeneration research. Curr Eye Res. 2015;40(5):501-9. doi: 10.3109/02713683.2014.935440 pmid: 24977298

31. Kurz DJ, Decary S, Hong Y, Erusalimsky JD. Senescence-associated (beta)-galactosidase reflects an increase in lysosomal mass during replicative ageing of human endothelial cells. J Cell Sci. 2000;113 (Pt 20):3613-22. pmid: 11017877

32. Reddy RK, Lalezary M, Kim SJ, Kammer JA, Kuchtey RW, Cherney EF, et al. Prospective Retinal and Optic Nerve Vitrectomy Evaluation (PROVE) study: findings at 3 months. Clinical Ophthalmology. 2013;7:1761-9. doi: 10.2147/OPTH.S49375 pmid: 24039396

33. Mastropasqua R, D’Aloisio R, Viggiano P, Borrelli E, Iafigliola C, Di Nicola M, et al. Early Retinal Flow Changes after Vitreoretinal Surgery in Idiopathic Epiretinal Membrane Using Swept Source Optical Coherence Tomography Angiography. Journal of Clinical Medicine. 2019;8(12):2067. doi: 10.3390/jcm8122067 pmid: 31771299

34. Romano MR, Cennamo G, Grassi P, Sparnelli F, Allegrini D, Cennamo G. Changes in macular pigment optical density after membrane peeling. PLoS One. 2018;13(5):e0197034. doi: 10.1371/journal.pone.0197034 pmid: 29758035

35. Fung AT, Galvin J, Tran T. Epiretinal membrane: A review. Clin Exp Ophthalmol. 2021;49(3):289-308. doi: 10.1111/ceo.13914 pmid: 33656784

36. Spaide R (2006). Etiology of late-age-related macular disease, in Age-related macular degeneration: A comprehensive textbook. pp. 23-39. D.V. Ilfara III, Liggett, P.V., Mieler, W.F, Quirox-Mercado, H., Jager, R.D., Tano, Y., Editor. Lippincot, Williams and Wilkins: Philadelphia. 